\title{
Endocrine disorders and the neurologic manifestations
}

\author{
Jeesuk Yu, MD, PhD \\ Department of Pediatrics, Dankook \\ University Hospital, Dankook \\ University College of Medicine, \\ Cheonan, Korea
}

\begin{abstract}
The nervous system and the endocrine system are closely interrelated and both involved intimately in maintaining homeostasis. Endocrine dysfunctions may lead to various neurologic manifestations such as headache, myopathy, and acute encephalopathy including coma. It is important to recognize the neurologic signs and symptoms caused by the endocrine disorders while managing endocrine disorders. This article provides an overview of the neurologic manifestations found in various endocrine disorders that affect pediatric patients. It is valuable to think about 'endocrine disorder' as a cause of the neurologic manifestations. Early diagnosis and treatment of hormonal imbalance can rapidly relieve the neurologic symptoms. Better understanding of the interaction between the endocrine system and the nervous system, combined with the knowledge about the pathophysiology of the neurologic manifestations presented in the endocrine disorders might allow earlier diagnosis and better treatment of the endocrine disorders.
\end{abstract}

\section{Key words: Endocrine system diseases, Neurologic manifestations, Child}

\section{Introduction}

Both the nervous system and the endocrine system are involved intimately in maintaining homeostasis. Therefore, endocrine dysfunctions may lead to various neurologic manifestations, which can occur in any endocrine disorders including disorders of pituitary gland, thyroid, parathyroids, pancreas, adrenal glands, and gonads. It is beneficial to know the neurologic signs and symptoms caused by the endocrine disorders in diagnosing as well as managing endocrine disorders.

This article provides an overview of the neurologic manifestations found in various endocrine disorders that affect pediatric patients. First of all, various neurologic symptoms found in endocrine disorders are covered in this article. Each neurologic manifestation is listed along with the possible endocrine disorders. Second, this article reviews about neurologic findings related to the endocrine disorders or their management.

\section{Hypothalamic-pituitary system}

It is important to understand the hypothalamic-pituitary system and the intimate relation between nervous system and endocrine system. The neuroendocrine system which is made up of the nervous system and the endocrine system work together to keep the body to function regularly. It focuses on the hypothalamic control to the secretion of pituitary hormones, but the broad concept includes multiple reciprocal interaction between the nervous system and the endocrine systems to maintain homeostasis and to respond properly to environmental stimuli through the regulated secretion of hormones, neurotransmitters, or neuromodulators ${ }^{1,2}$. Neurons release their neurotransmitters and neuromodulators at synapses. Neurosecretory cells secrete substances directly into the bloodstream to act as hormones. They include neurohypophyseal and hypophysiotropic cells. Hypothalamus is the ultimate brain structure involved in maintaining homeostasis. It has many specified nuclei which receive sensory
Address for correspondence: Jeesuk Yu, MD, PhD

Department of Pediatrics, Dankook University Hospital, Dankook

University College of Medicine, 201 Manghyang-ro, Dongnam-gu, Cheonan 330-715, Korea

Tel: $+82-41-550-6590$

Fax: +82-41-565-6167

E-mail:dryujs@dankook.ac.kr 
inputs from the external and internal environment such as light, nociception, temperature, blood pressure, blood osmolality, and blood glucose levels ${ }^{1,2)}$. Many hormones also exert both negative and positive feedback directly on the hypothalamus. Hypothalamus provides coordinated responses to the pituitary glands, cerebral cortex, brain stem and spinal cord, and sympathetic and parasympathetic preganglionic neurons to maintain homeostasis through the coordinated endocrine, behavioral, and autonomic responses ${ }^{1,2)}$.

\section{Potential endocrine disorders according to the specific neurologic manifestations}

Various neurologic signs and symptoms arising from the endocrine disorders include headache, altered state of consciousness, abnormal muscle strength, muscle tone, muscle stiffness and cramps, movement disorders, and psychomotor retardation $^{3)}$.

\section{Headache}

Headache may be a nonspecific sign, but it can be caused by pathologic conditions including idiopathic intracranial hypertension $^{3)}$.

Idiopathic intracranial hypertension (pseudotumor cerebri syndrome, PTCS) is the presence of elevated intracranial pressure in the setting of normal brain parenchyma and cerebrospinal fluid $^{4)}$. Headache, vomiting, vision changes, abducens nerve palsy, and papilledema are commonly presented $^{3)}$. Only irritability, somnolence, or apathy may be found in young children ${ }^{3)}$. If it is untreated, it may progress to optic atrophy and vision will be lost rapidly ${ }^{3)}$. Therefore, early diagnosis and treatment are crucial. The exact mechanism of PTCS is unclear, but it may occur associated with a variety of conditions, including various endocrine disorders such as adrenal insufficiency, diabetic ketoacidosis on treatment, hyperadrenalism, hyperthyroidism, and hypoparathyroidism ${ }^{3)}$. Sheldon et al. ${ }^{4}$ presented that pediatric PTCS is a neuroendocrine disorder, illustrating many metabolic and hormonal derangements within the hypothalamic-pituitary-adrenal axis, renin-angiotensin-aldosterone, growth hormone $(\mathrm{GH})$ and insulin-like growth factor-1 (IGF-1), hypothalamic-pituitarythyroid axis, hypothalamic-pituitary-gonadal axis, and the posterior pituitary and antidiuretic hormone.

\section{Altered mentality}

Altered mental status is a common presentation in the emergency department, and can be caused by endocrine emergencies ${ }^{5}$. Attention, awareness, and consciousness can be maintained by the interaction among the brainstem reticular core, the thalamus, and the cerebral cortex ${ }^{6}$. Impaired consciousness means a significant alteration in the wakefulness and the awareness of self and of the environment ${ }^{7)}$. It is important to find the underlying causes and to promptly stabilize the vital signs ${ }^{5,8)}$. The causes of coma in children can be classified with infectious or inflammatory origin, structural lesions, and metabolic, toxic or nutritional conditions ${ }^{8)}$. Metabolic or endocrine encephalopathy should be considered as a differential diagnosis in the patients presenting no focal neurologic signs and no meningeal irritation signs ${ }^{8}$.

Diabetic coma is one of differential diagnosis in the emergency room in managing patients with altered mentality. Diabetic ketoacidosis (DKA) and hyperglycemic hyperosmolar syndrome are the most serious acute hyperglycemic emergency $^{9-11)}$. Hypoglycemia, cerebral edema following the treatment of DKA may also be presented with altered mentality ranging from general weakness, lethargy, irritability to coma ${ }^{8)}$. Hyponatremic hypovolemic adrenal crisis in the patients with underlying congenital adrenal hyperplasia (CAH), adrenal hypoplasia congenita, familial glucocorticoid deficiency, or adrenocorticotropic hormone (ACTH) deficiency can lead to altered mentality ${ }^{8)}$. Hypocalcemic tetany or seizure with or without underlying hypoparathyroidism may also result in impaired consciousness ${ }^{3)}$. It is noteworthy to remember that apathy, delirium, or psychosis can happen at serum calcium concentrations greater than $11 \mathrm{mg} / \mathrm{dL}^{3)}$. Checking the level of blood glucose, calcium and electrolytes should be considered in the first step of evaluating altered mentality.

Severe hyperthyroidism and hypothyroidism may be the rare cause of altered mentality, thyroid storm and hypothyroid coma, respectively. They could be fatal if not treated properly ${ }^{12)}$. Behavioral and cognitive changes, myoclonus, seizures, psychosis, involuntary movements, and even coma may occur. In hypothyroid coma, there may be a history of previous thyroid disease and progressive lethargy with hypothermia, bradycardia, constipation, dyspnea, yellow and dry skin, large tongue, and rarely seizure. In thyroid storm, fever, tachyarrhythmia, tachypnea, dyspnea, congestive heart failure, diarrhea, nausea, vomiting, and hyperhidrosis can be manifested. In addition, tremors, anxiety, confusion, delirium up to comatose state may develop. As the precipitating factors, major surgery, trauma, systemic infections, severe emotional stress, or diabetic ketoacidosis may be considered. Therefore, thyroid function should be evaluated in the management of diabetic ketoacidosis. It was reported that thyroid storm was presented as cardiorespiratory distress in a 2-year-old girl referred for urgent lung perfusion imaging, which suggests the importance of suspicion for thyroid disease in those who show unstable vital signs ${ }^{13)}$. Hashimoto's encephalopathy can be developed in the patients with autoimmune thyroid diseases and is characterized by a steroid-responsive encephalopathy with the presence of antithyroid antibodies ${ }^{14-16)}$.

\section{Abnormal muscle strength, muscle tone and gait}

Muscle weakness, pain, and stiffness are common symptoms of endocrine disorders ${ }^{3)}$. Systemic characteristic symptoms of specific endocrine disorders usually precede the onset of 
weakness, but muscle weakness may be the initial symptom ${ }^{3)}$. Endocrine myopathy should be considered as one of the etiology of muscle weakness, because specific treatment is available in endocrine myopathy ${ }^{177}$.

Thyroid dysfunction (hyper- or hypothyroidism), parathyroid disorders (hyper- or hypoparathyroidism), and adrenal diseases (Cushing disease, Addison disease, or hyperaldosteronism) may cause endocrine myopathies ${ }^{3,17,18)}$. Kruse ${ }^{18)}$ pointed that clinical features of most of endocrine myopathies in childhood are usually characterized by the presence of proximal muscle involvement such as pelvic or shoulder girdle muscles, relatively mild morphological muscular abnormalities even in the presence of severe clinical symptoms, and favorable outcome to treatment. Weakness is usually much more prominent in the legs than in the arms, and abnormal gait can be the initial symptom of either proximal or distal leg weakness ${ }^{3)}$. A case of subclinical hypothyroidism presenting with gait abnormality was reported in an old $\operatorname{man}^{19)}$. Electrolyte imbalances such as hyper- or hyponatremia, hyper- or hypokalemia, hypophosphatemia, hypocalcemia, and hypomagnesemia can all be the cause of myopathies accompanied with endocrine disorders ${ }^{3,17}$. Deep tendon reflexes may be normal or diminished but generally not absent. The serum creatine kinase is usually normal. However it can be elevated which does not correlate with the severity of muscle weakness ${ }^{3)}$.

A cramp, an involuntary painful contraction of a muscle or a part of a muscle, can occur in normal children associated with vigorous exercise, but it can be caused by endocrine disorders including adrenal insufficiency, hypothyroidism or hyperthyroidism ${ }^{3)}$. Muscle stiffness and spasms occur in myotonia, dystonia, and other movement disorders, but can be present in hypothyroidism or thyrotoxicosis when motor unit activity is continuous ${ }^{3)}$. In hypothyroidism, the stiffness gets worsen by activity and may be painful with the slowing of muscular contraction and relaxation in performing tendon reflexes.

Tone is functionally defined as resistance to passive movement. Therefore, hypotonia is indicated to decreasing resistance to passive movement ${ }^{3}$. Profound hypotonia with obesity and variable degree of intellectual disability may suggest PraderWilli syndrome (PWS ${ }^{20)}$, and hypothyroidism is one of considerable endocrine disease presenting hypotonia ${ }^{21)}$. Webb et al. ${ }^{22)}$ reported neuromuscular dysfunction in the adults with GH deficiency (GHD) associated with fatigue, tiredness and myalgia using the muscle biopsy and the neurophysiological study. Schweizer et al ${ }^{23)}$ described that supraphysiological doses of GH treatment to the short children with small for gestational age (SGA) led to a concomitant increment in height, muscle mass, and function with a decrease in fat mass. Reus et al. ${ }^{24)}$ presented that GH treatment increased muscle thickness in infants with PWS, which was related to muscle strength and motor development.

\section{Movement disorders}

Movement disorders are the disorders causing involuntary movements such as chorea, athetosis or tremor ${ }^{3)}$. Many abnormal movements are paroxysmal or intermittent ${ }^{3)}$. Chorea, a rapid repetitive movement affecting any part of the body, is neither rhythmic nor stereotyped and can occur in hyperthyroidism ${ }^{3)}$. Athetosis, a slow and writhing movement of the limbs, is often associated with chorea. Choreoathetosis can occur in hyperthyroidism, Addison disease, hypernatremia, hypocalcemia and hypoparathyrodism ${ }^{3,37)}$. Tremor, an involuntary oscillating movement with a fixed frequency, may occur physiologically but hyperthyroidism should be considered as a potential cause $e^{3)}$.

\section{Developmental delay}

The effect of GHD on brain structure, motor function, and cognition were studied by cognitive assessment and diffusion tensor, and volumetric magnetic resonance imaging (MRI) in children with GHD. In GHD, lower cognitive scales, white matter abnormalities in the corpus callosum and corticospinal tract, and reduced volumes in thalamus, hippocampus, and globus pallidus were found compared with controls with idiopathic short stature ${ }^{25)}$.

In the review of thirty Korean patients diagnosed as PWS, more than two years of GH treatment in 14 PWS infants and toddlers resulted in increasing head circumference-standard deviation score (SDS), IGF-1 SDS, IGF binding protein-3 SDS, lean body mass, and bone mineral content and showed an improvement in motor development ${ }^{26)}$.

Thyroid hormone regulates neuronal migration, differentiation, and myelination including cerebellar development ${ }^{27)}$. Transgenic mouse models showed that thyroid gland dysgenesis or decreasing levels of thyroid hormones in brain tissue was associated with cerebellar phenotypes such as ataxia, impaired balance and coordination, and reduced locomotor activity $^{27)}$.

Congenital hypothyroidism resulting from thyroid dysgenesis or dyshormonogenesis, occurring in 1 per 3,000-4,000 live births, may present delayed development, if not treated promptly. Early diagnosis and replacement of thyroid hormone are critical for a favorable outcome. Owing to the neonatal newborn screening program, almost all patients with congenital hypothyroidism can be detected and treated early in newborn period in Korea. It may be beneficial considering the genetic causes of congenital hypothyroidism such as MCT8 mutations (Allan-Herndon-Dudley syndrome) in the evaluation of floppy male infants with development delay, especially if the male has no history of perinatal asphyxia ${ }^{21,28}$. Rodrigues et al. ${ }^{21)}$ emphasized that the simple evaluation of triiodothyronine, thyroxine, and thyroid-stimulating hormone levels can guide the diagnosis, avoiding a number of invasive and expensive investigations and allowing appropriate genetic counseling to 
the families.

Recently several reports describing the association between various adrenal disorders and neurologic manifestations were published ${ }^{29-32}$. Childhood Cushing syndrome can be associated with cognitive impairment and behavioral abnormality, even after recovery from the hypercortisolism ${ }^{29}$. Exposure to excessive replacement of glucocorticoids in children during the treatment of adrenal insufficiency has been associated with neurological and MRI abnormalities including delayed myelination and brain atrophy ${ }^{29)}$. Even though the clinical implication is unclear, several reports suggest that patients with $\mathrm{CAH}$ are more likely to have white matter abnormalities, temporal lobe atrophy or hippocampal dysgenesis probably produced by the disease and its treatment. Mental retardation, tremor, asymmetric tendon reflexes, and cerebellar syndrome were found in some patients with $\mathrm{CAH}^{30,31)}$.

Ghetti et al. ${ }^{33)}$ showed that children with DKA history had significantly lower rates of accurate memory on both the color and the spatial tasks compared with children without DKA history. They concluded that DKA disrupts memory function and emphasized the importance of DKA prevention.

\section{Neurologic findings in specific endocrine disorders}

\section{Hypothalamic-pituitary dysfunction}

It is crucial to diagnose various hypothalamic-pituitary lesions as early as possible. Endocrine symptoms often precede neuro-ophthalmic symptoms. In a retrospective, single-center, cohort study of 176 patients aged 6 years (range, 0.2-18 years), with hypothalamic-pituitary lesions including craniopharyngioma, optic pathway glioma, suprasellar arachnoid cyst, hamartoma, germ cell tumor, and hypothalamicpituitary astrocytoma, abnormal body mass index or decreased growth velocity occurred in two-thirds of patients before the presentation of neuro-ophthalmic symptoms ${ }^{34)}$. This suggests the need for more cautious approach to identify the etiology of endocrine symptoms for earlier diagnosis of hypothalamicpituitary lesions ${ }^{34)}$.

Acute endocrine changes are commonly found after traumatic brain injury in children, including changes in hypothalamicpituitary-adrenal axis and antidiuretic hormone production and releas ${ }^{35,36)}$. In the long term, there are both temporary and permanent alterations in pituitary function such as GHD, pubertal disorders, ACTH deficiency, diabetes insipidus, central hypothyroidism, and elevated prolactin ${ }^{35)}$. The authors emphasized routine monitoring at least 1 year after injury for early detection of hormonal imbalance to permit normal growth and development ${ }^{35}$.

\section{Thyroid disorders}

In congenital hypothyroidism, mental retardation, hypo- tonia, constipation, somnolence, apnea, large fontanels, and sensorineural hearing loss may be presented ${ }^{17,37)}$. It may accompany Kocher-Debre-Semelaigne syndrome which is characterized by generalized muscular hypertrophy, predominating in the calf muscles associated with hypothyroidism ${ }^{38}$. Myopathy of hypothyroidism in infancy and childhood is featured by lower extremity or generalized muscular hypertrophy, myxedema, and short stature. Although it is possible to detect congenital hypothyroidism early in neonate through neonatal screening test, it can rarely be missed, requiring repeated thyroid function test at any time if there are suspicious clinical symptoms suggesting hypothyroidism. In neonatal hyperthyroidism, craniosynostosis and developmental delay can occur.

Acquired hypothyroidism can accompany psychosis, seizures, ataxia, and coma ${ }^{12,37)}$. Immune-mediated mechanisms and vasculitis accompanying cerebral hypoperfusion may result in Hashimoto's encephalopathy. Hashimoto's encephalopathy is not common in childhood, but favorable outcome can be expected with steroid or immune treatment ${ }^{14-16)}$.

Graves' disease presents neurologic features such as emotional instability, anxiety, irritability, restlessness, and inattentiveness. Myopathy including muscular weakness or cramp, ocular symptoms such as lid lag, impairment of convergence, or ophthalmoplegia, and movement disorders such as tremor or chorea can be manifested. Thyrotoxic periodic paralysis or myasthenia gravis rarely occur in hyperthyroidism ${ }^{17,37)}$. Severe thyrotoxicosis may result in a change in consciousness, as seen in thyroid storm ${ }^{12)}$.

\section{Parathyroid disorders}

Neurologic manifestations associated with parathyroid disorders are related with serum calcium levels. Hypoparathyroidism may result in hypocalcemia causing muscular pain, cramp, numbness, stiffness, tetany, apnea, and seizures. In addition, increased intracranial pressure with headaches and vomiting may occur ${ }^{17,37,39)}$. Autoimmune polyendocrine syndrome type 1 (APS1) was reported in two siblings who presented hypocalcemic seizure followed by hypoparathyroidism ${ }^{40)}$.

Hyperparathyroidism may cause hypercalcemia which leads to muscular weakness, anorexia, nausea, vomiting, and encephalopathy ${ }^{17,37,39)}$. Polydipsia, polyuria, weight loss, headache, seizure, and psychiatric symptoms may occur in this condition ${ }^{17,37,39)}$. Although primary hyperparathyroidism is not common in children, severe central nervous system and metabolic derangement may develop. Therefore, cautious interpretation of hypercalcemia and prompt diagnosis are necessary to prevent it ${ }^{41)}$.

\section{Adrenal disorders}

Primary adrenal insufficiency includes CAH, APS, ACTH resistance, adrenoleukodystrophy, adrenal hypoplasia conge- 
nita, adrenal hemorrhage, and IMAGe (intrauterine growth restriction, metaphyseal dysplasia, adrenal hypoplasia congenita, and genital anomalies) syndrome ${ }^{42,43)}$. Hypotension, hyperpigmentation, hyponatremia, hyperkalemia, and hypoglycemia are presenting signs. Hyperkalemia may not be found in primary adrenal insufficiency in childhood, thus the absence of hyperkalemia cannot rule out this condition ${ }^{42)}$. Adrenal insufficiency should be suspected in the presence of a combination of chronic or subacute clinical symptoms including chronic fatigue, muscle weakness, anorexia, nausea, vomiting, diarrhea, hypotension, weight loss, headache and skin hyperpigmentation ${ }^{42-44)}$. Serum ACTH, aldosterone or plasma renin activity can be included in the initial step evaluating suspected primary adrenal insufficiency.

Absolute and relative adrenal insufficiency may occur in critically ill children with systemic inflammatory response syndrome or sepsis ${ }^{45-47)}$. In the state of catecholamine-resistant shock, it is important to check whether there is adrenal insufficiency, and ACTH stimulation test may help to the $\operatorname{diagnosis}^{44-46)}$.

Excessive glucocorticoids in primary or secondary Cushing syndrome may accompany headache, proximal muscle weakness, cognitive deficits, and behavioral disturbances ${ }^{3,17,29)}$. In hyperaldosteronism, weakness from hypokalemia can be manifested. Primary aldosteronism and pheochromocytoma should be included in the differential diagnosis in a case with hypertension $^{17)}$.

\section{Diabetes mellitus}

Diabetic ketoacidosis and cerebral edema may be presented with various neurologic manifestations ${ }^{48,49)}$. Agitation, confusion, lethargy, headache, and vomiting can be presented with cerebral edema. For the prevention of the development of cerebral edema, gradual rehydration over 48 hours, avoidance of hypotonic fluids, cautious use of bicarbonate, and careful correction of hyperglycemia seem to be beneficial. If cerebral edema is suspected, rapid infusion of mannitol, head elevation, fluid restriction, and hyperventilation should be done as soon as possible.

In hypoglycemia, various neurologic manifestations such as tremor, confusion, behavioral changes, blurred vision, seizure, and coma may develop.

Although neurological deterioration in children with DKA is commonly caused by cerebral edema, stroke can develop because children with hyperglycemia and DKA are prone to thrombosis $^{50)}$. It may be beneficial to suspect the possibility of the development of thrombosis, infection or hemorrhage when focal neurological deficits are apparent or neurologically deteriorated in the patients with $\mathrm{DKA}^{50,51)}$.

Symptomatic diabetic neuropathy (DNP) in childhood is not common, but subclinical DNP is common among children and adolescents with at least 5-year duration of type 1 diabetes mellitus ${ }^{52)}$. Even though clinical DNP is uncommon in children and adolescents, it can be detected through neurophysiological studies.

\section{Conclusions}

Endocrine disorders can be manifested by various neurologic symptoms and signs ranging from headache, myopathy to acute encephalopathy including coma. It is valuable to think about 'endocrine disorder' as the cause of the neurologic impairment. Early diagnosis and treatment of hormonal imbalance may rapidly relieve the neurologic symptoms. Better understanding of the interaction between the endocrine system and the nervous system, combined with the knowledge about the pathophysiology of the neurologic symptoms and signs presented in the endocrine disorders might allow earlier diagnosis and better treatment of the endocrine disorders.

\section{Conflict of interest}

No potential conflict of interest relevant to this article was reported.

\section{References}

1. Low MJ. Neuroendocrinology. In: Melmed S, Polonsky KS, Larsen PR, Kronenberg HM, editors. Williams textbook of endocrinology. 12th ed. Philadelphia: Elsevier/Saunders, 2011:103-74.

2. Parks JS, Felner EI. Hormones of the hypothalamus and pituitary. In: Kliegman RM, Stanton BF, St Geme JW, Schor NF, Behrman RE. editors. Nelson textbook of pediatrics. 19th ed. Philadelphia: Elsevier/Saunders, 2011:1876.

3. Fenichel GM. Clinical pediatric neurology: a signs and symptoms approach. 4th ed. Philadelphia: W.B. Saunders, 2001.

4. Sheldon CA, Kwon YJ, Liu GT, McCormack SE. An integrated mechanism of pediatric pseudotumor cerebri syndrome: evidence of bioenergetic and hormonal regulation of cerebrospinal fluid dynamics. Pediatr Res 2014 Nov 24 [Epub]. http://dx.doi:10.1038/pr.2014.188.

5. Park E, Abraham MK. Altered mental status and endocrine diseases. Emerg Med Clin North Am 2014;32:367-78.

6. Boveroux P, Bonhomme V, Boly M, Vanhaudenhuyse A, Maquet P, Laureys S. Brain function in physiologically, pharmacologically, and pathologically altered states of consciousness. Int Anesthesiol Clin 2008;46:131-46.

7. Taylor DA, Ashwal S. Impairment of consciousness and coma. In: Swaiman KF, Ashwal S, Ferriero DM, Schor NF. Swaiman's pediatric neurology. 5th ed. [Edinburgh]: Elsevier Saunders, 2012:1063-7.

8. Sharma S, Kochar GS, Sankhyan N, Gulati S. Approach to the child with coma. Indian J Pediatr 2010;77:1279-87.

9. Kitabchi AE, Umpierrez GE, Fisher JN, Murphy MB, Stentz FB. Thirty years of personal experience in hyperglycemic 
crises: diabetic ketoacidosis and hyperglycemic hyperosmolar state. J Clin Endocrinol Metab 2008;93:1541-52.

10. Nyenwe EA, Kitabchi AE. Evidence-based management of hyperglycemic emergencies in diabetes mellitus. Diabetes Res Clin Pract 2011;94:340-51.

11. Zeitler P, Haqq A, Rosenbloom A, Glaser N; Drugs and Therapeutics Committee of the Lawson Wilkins Pediatric Endocrine Society. Hyperglycemic hyperosmolar syndrome in children: pathophysiological considerations and suggested guidelines for treatment. J Pediatr 2011;158:9-14, 14.e1-2.

12. Papi G, Corsello SM, Pontecorvi A. Clinical concepts on thyroid emergencies. Front Endocrinol (Lausanne) 2014;5:102.

13. Arsos G. Unexpected diagnosis of thyroid storm in a young child referred for urgent lung perfusion imaging. Clin Nucl Med 2013;38:661-3.

14. Vasconcellos E, Pina-Garza JE, Fakhoury T, Fenichel GM. Pediatric manifestations of Hashimoto's encephalopathy. Pediatr Neurol 1999;20:394-8.

15. Yu HJ, Lee J, Seo DW, Lee M. Clinical manifestations and treatment response of steroid in pediatric Hashimoto encephalopathy. J Child Neurol 2013;29:938-42.

16. Chang JS, Chang TC. Hashimoto's encephalopathy: report of three cases. J Formos Med Assoc 2014;113:862-6.

17. Sharma V, Borah P, Basumatary LJ, Das M, Goswami M, Kayal AK. Myopathies of endocrine disorders: a prospective clinical and biochemical study. Ann Indian Acad Neurol 2014;17:298-302.

18. Kruse K. Myopathies in endocrine disorders. Monatsschr Kinderheilkd 1984;132:581-6.

19. Edvardsson B, Persson S. Subclinical hypothyroidism presenting with gait abnormality. Neurologist 2010;16:1156.

20. Bridges $\mathrm{N}$. What is the value of growth hormone therapy in Prader Willi syndrome? Arch Dis Child 2014;99:166-70.

21. Rodrigues F, Grenha J, Ortez C, Nascimento A, Morte B, M-Belinchón M, et al. Hypotonic male infant and MCT8 deficiency- a diagnosis to think about. BMC Pediatr 2014;14:252.

22. Webb SM, de Andres-Aguayo I, Rojas-Garcia R, Ortega E, Gallardo E, Mestron A, et al. Neuromuscular dysfunction in adult growth hormone deficiency. Clin Endocrinol (Oxf) 2003;59:450-8.

23. Schweizer R, Martin DD, Schonau E, Ranke MB. Muscle function improves during growth hormone therapy in short children born small for gestational age: results of a peripheral quantitative computed tomography study on body composition. J Clin Endocrinol Metab 2008;93:297883.

24. Reus L, Pillen S, Pelzer BJ, van Alfen-van der Velden JA, Hokken-Koelega AC, Zwarts M, et al. Growth hormone therapy, muscle thickness, and motor development in Prader-Willi syndrome: an RCT. Pediatrics 2014;134:e161927.

25. Webb EA, O'Reilly MA, Clayden JD, Seunarine KK, Chong
WK, Dale N, et al. Effect of growth hormone deficiency on brain structure, motor function and cognition. Brain 2012;135(Pt 1):216-27.

26. Kim YJ, Cheon CK. Prader-Willi syndrome: a single center's experience in Korea. Korean J Pediatr 2014;57:310-6.

27. Faustino LC, Ortiga-Carvalho TM. Thyroid hormone role on cerebellar development and maintenance: a perspective based on transgenic mouse models. Front Endocrinol (Lausanne) 2014;5:75.

28. Piana RL, Vanasse M, Brais B, Bernard G. Myelination delay and Allan-Herndon-Dudley syndrome caused by a novel mutation in the SLC16A2 gene. J Child Neurol 2014 Nov 7 [Epub].http://dx.doi.org/10.1177/0883073814555189.

29. Salpietro V, Polizzi A, Di Rosa G, Romeo AC, Dipasquale V, Morabito P, et al. Adrenal disorders and the paediatric brain: pathophysiological considerations and clinical implications. Int J Endocrinol 2014;2014:282489.

30. Bergamaschi R, Livieri C, Uggetti C, Candeloro E, Egitto MG, Pichiecchio A, et al. Brain white matter impairment in congenital adrenal hyperplasia. Arch Neurol 2006;63:4136.

31. Mnif MF, Kamoun M, Mnif F, Charfi N, Kallel N, Rekik N, et al. Brain magnetic resonance imaging findings in adult patients with congenital adrenal hyperplasia: increased frequency of white matter impairment and temporal lobe structures dysgenesis. Indian J Endocrinol Metab 2013;17:121-7.

32. Nass R, Heier L, Moshang T, Oberfield S, George A, New MI, et al. Magnetic resonance imaging in the congenital adrenal hyperplasia population: increased frequency of white-matter abnormalities and temporal lobe atrophy. J Child Neurol 1997;12:181-6.

33. Ghetti S, Lee JK, Sims CE, Demaster DM, Glaser NS. Diabetic ketoacidosis and memory dysfunction in children with type 1 diabetes. J Pediatr 2010;156:109-14.

34. Taylor M, Couto-Silva AC, Adan L, Trivin C, Sainte-Rose C, Zerah M, et al. Hypothalamic-pituitary lesions in pediatric patients: endocrine symptoms often precede neuroophthalmic presenting symptoms. J Pediatr 2012;161:85563.

35. Rose SR, Auble BA. Endocrine changes after pediatric traumatic brain injury. Pituitary 2012;15:267-75.

36. Acerini CL, Tasker RC, Bellone S, Bona G, Thompson CJ, Savage MO. Hypopituitarism in childhood and adolescence following traumatic brain injury: the case for prospective endocrine investigation. Eur J Endocrinol 2006;155:663-9.

37. Tonner DR, Schlechte JA. Neurologic complications of thyroid and parathyroid disease. Med Clin North Am 1993;77:251-63.

38. Shaw C, Shaw P. Kocher-Debre-Semelaigne syndrome: hypothyroid muscular pseudohypertrophy-a rare report of two cases. Case Rep Endocrinol 2012;2012:153143.

39. Agrawal L, Habib Z, Emanuele NV. Neurologic disorders of mineral metabolism and parathyroid disease. Handb Clin Neurol 2014;120:737-48.

40. Eyal O, Oren A, Juppner H, Somech R, De Bellis A, 
Mannstadt M, et al. Hypoparathyroidism and central diabetes insipidus: in search of the link. Eur J Pediatr 2014;173:1731-4.

41. Damiani D, Aguiar CH, Bueno VS, Montenegro FL, Koch VH, Cocozza AM, et al. Primary hyperparathyroidism in children: patient report and review of the literature. J Pediatr Endocrinol Metab 1998;11:83-6.

42. Hsieh $S$, White PC. Presentation of primary adrenal insufficiency in childhood. J Clin Endocrinol Metab 2011;96:E925-8.

43. Choudhary S, Alam A, Dewan V, Yadav D, Dubey NK. An unusual presentation of Addison's disease: a case report. Clin Pediatr Endocrinol 2011;20:57-60.

44. Hinz LE, Kline GA, Dias VC. Addison's disease in evolution: an illustrative case and literature review. Endocr Pract 2014;20:e176-9.

45. Hebbar KB, Stockwell JA, Leong T, Fortenberry JD. Incidence of adrenal insufficiency and impact of corticosteroid supplementation in critically ill children with systemic inflammatory syndrome and vasopressordependent shock. Crit Care Med 2011;39:1145-50.

46. Menon K, Ward RE, Lawson ML, Gaboury I, Hutchison JS, Hébert PC, et al. A prospective multicenter study of adrenal function in critically ill children. Am J Respir Crit Care
Med 2010;182:246-51.

47. Santos L. Stress response in critical illness. Curr Probl Pediatr Adolesc Health Care 2013;43:264-72.

48. Gunst J, Van den Berghe G. Blood glucose control in the intensive care unit: benefits and risks. Semin Dial 2010;23:157-62.

49. Pasquel FJ, Umpierrez GE. Hyperosmolar hyperglycemic state: a historic review of the clinical presentation, diagnosis, and treatment. Diabetes Care 2014;37:3124-31.

50. Ho J, Mah JK, Hill MD, Pacaud D. Pediatric stroke associated with new onset type 1 diabetes mellitus: case reports and review of the literature. Pediatr Diabetes 2006;7:116-21.

51. Mulder L, Onur O, Kleis L, Borders H, Cemeroglu AP. Atypical neurologic presentations of new onset type 1 diabetes mellitus in pediatric age group: a report of five unusual cases and review of the literature. J Pediatr Endocrinol Metab 2014;27:749-56.

52. Nelson D, Mah JK, Adams C, Hui S, Crawford S, Darwish $\mathrm{H}$, et al. Comparison of conventional and non-invasive techniques for the early identification of diabetic neuropathy in children and adolescents with type 1 diabetes. Pediatr Diabetes 2006;7:305-10. 